\title{
Introducing a multifaceted approach to the management of diabetes mellitus in resource-limited settings
}

\begin{abstract}
S Pillay, C Aldous
Somasundram Pillay is a specialist physician working at Edendale Hospital, Pietermaritzburg, South Africa, and involved in both undergraduate and postgraduate teaching and supervision. Upon completion of his fellowship exams, his research focus has centred around improving diabetes care at a regional level hospital. He has completed his MMed and is now working on his PhD dissertation. His main interest lies in diabetes and endocrinology. Colleen Aldous is Associate Professor and Academic Leader for Research in the School of Clinical Medicine at the University of KwaZulu-Natal, Durban, South Africa. She is a medical scientist with a PhD in science education and is involved in postgraduate research mentorship across several medical disciplines including surgery, orthopaedics, dermatology, paediatrics, ophthalmology, general medicine and psychology. Her own research interest is human genetics.
\end{abstract}

Corresponding author: S Pillay (drspillay@iafrica.com)

Globally diabetes mellitus (DM) and its complications are placing an enormous burden on individual patients and countries alike. South Africa is a developing country already under enormous pressure from communicable diseases such as HIV and tuberculosis. Added to this is DM, which serves to fuel the interactions between communicable and non-communicable diseases. Data from KwaZulu-Natal Province (KZN) have demonstrated that the majority of patients with DM in the public healthcare sector are diagnosed and started on treatment at their local resource-limited healthcare clinics. This article describes introduction of a multifaceted approach to the management of DM in a resource-limited clinic at Edendale Hospital, Pietermaritzburg, KZN. Strategies like this may help provide a blueprint for other resourcelimited healthcare facilities in developing countries.

S Afr Med J 2016;106(5):456-458. DOI:10.7196/SAMJ.2016.v106i5.10409

Diabetes mellitus (DM) and its complications remain a health threat to individual patients and a burden to the health economies of countries all over the world, particularly developing countries that are already overburdened with communicable diseases (CDs) such as HIV and tuberculosis (TB). South Africa (SA) is one such developing country, where DM can serve to fuel the interactions between $\mathrm{CDs}$ and non-communicable diseases (NCDs). HIV infection increases the risk of developing type 2 DM two-fold, while DM increases the risk of contracting TB three-fold. ${ }^{[1]}$ SA has the third-highest prevalence of TB globally. ${ }^{[2]}$ Improving diabetes control may provide a means of breaking this interaction between CDs and NCDs.

The factors impeding attainment of optimal diabetes control include obesity and healthcare worker compliance with clinical guidelines. The prevalence of type $2 \mathrm{DM}$ is being fuelled by the obesity pandemic, with SA having the highest rate of obesity among females in sub-Saharan Africa. ${ }^{[3]}$ Optimal control of DM is not being achieved in either the private or the public sector in SA..$^{[4-5]}$ Healthcare worker compliance with clinical guidelines has been shown to be suboptimal both in SA and globally. ${ }^{[6-8]}$

The following interventions have demonstrated improvements in diabetes care:

- Electronic patient registries have been shown to improve glycaemic and low-density lipoprotein and total cholesterol control in resource-limited settings. ${ }^{[9]}$

- A study by Ricci-Cabello et al. ${ }^{[10]}$ suggests that multiple intervention strategies directed at both clinicians and patients can improve diabetes control in resource-limited clinics.

We have shown ${ }^{[11]}$ that the majority of patients with DM attending public sector healthcare facilities in KwaZulu-Natal Province (KZN), SA, over the period 2010 - 2014 were diagnosed and initiated on diabetes treatment at their clinics rather than at hospital level. Multifaceted strategies targeted at clinic level could therefore provide an ideal approach. With most diabetes clinics in developing countries being resource-limited, use of the scarce resources available needs to be maximised to achieve some degree of control of this pandemic.

\section{As things were...}

Edendale Hospital is a busy regional hospital in Pietermaritzburg, the capital of KZN, which provides district as well as regional services. The diabetes clinic in this resource-limited hospital originally mirrored what exists in many other developing countries. Problems at the clinic included an unstructured booking system, which meant that a large number of patients seen did not in fact require specialist care at that point, clinician understaffing, poor or no patient education and staff in-service training, lack of the equipment necessary for appropriate examination of the patient with DM, an incomplete multidisciplinary team (lacking podiatrist, dietician and regular ophthalmological assessments), limited access to selfmonitoring of blood glucose (SMBG), and no means of collecting and auditing patient data.

\section{Changes made at the Edendale}

Hospital diabetes clinic

Acknowledging the shortage of resources and having assessed the deficits, the following changes were made.

\section{Organisation of the clinic}

- A structured booking system was introduced in the clinic. Once patients had been appropriately down-referred to their local community healthcare clinics after being stabilised, the number of patients seen at the Edendale clinic decreased from $60-70$ a week to a more manageable $30-40$. 
Staffing and patient education

- A multidisciplinary team was established that included the following members:

- specialist physician

- family physician

- medical officers/interns

- nursing staff

- diabetes nurse-educator

- dieticians

- podiatrist

- ophthalmologist for annual eye assessments.

All the members of this team were involved in intensive patient education. Patient diabetes educational material was acquired and displayed strategically in waiting areas.

- A diabetes nurse-educator was employed to oversee all clinic bookings and the general weekly running of the clinic. All the other members of the team were employed at Edendale Hospital and allocated to the clinic weekly.

\section{Staff training on all aspects of the care and needs of the patient with DM}

- Nursing staff working at the clinic were trained on all aspects of diabetes care by the specialist physicians.

- All clinicians working at the clinic were re-trained on the management of diabetes using the 2012 South African diabetes guidelines. ${ }^{[12]}$ Laminated copies of these guidelines were affixed to the walls of each consulting room.

\section{Data collection tools}

Two data collection tools were designed and introduced into the clinic.

- An ink-based stamp was used to stamp outpatient (OP) files of all patients visiting the clinic. It ensured that the clinical variables listed below were routinely measured for every patient and entered directly into the patient's OP folder. This decreased the risk of these values being written on loose pieces of paper, which are easily mislaid. The vital examinations that needed to be completed by the nursing staff before the patient's consultation with the clinician were listed on the stamp as follows:

- sitting and standing blood pressure $(\mathrm{mmHg})$

- resting pulse rate $(/ \mathrm{min})$

- height $(\mathrm{cm})$

- weight $(\mathrm{kg})$

- body mass index (BMI) $\left(\mathrm{kg} / \mathrm{m}^{2}\right)$

- waist circumference $(\mathrm{cm})$

- urine dipstick findings (all patients visiting the clinic now had a urine dipstick examination performed on every visit)

- random blood glucose (RBG) ( $\mathrm{mmol} / \mathrm{L})$.

- A specialised comprehensive datasheet was completed in triplicate by the attending clinician and served the following purposes:

- To ensure standardisation in the evaluation and management of all patients seen at the clinic

- To enable communication between the clinic and other healthcare facilities and personnel that the patient might consult at a later stage

- To ensure that commonly forgotten areas of diabetes management are reinforced (e.g. the need for regular ophthalmological and foot care and dietician reviews)

- To facilitate auditing and reporting.

A computer program that matched the datasheet was designed for the clinic, and all the data from the datasheets were captured on it. This program was written using Visual Basic Studio 2010 and
.NET Framework 4 technologies (Microsoft, USA) Reports were then generated via Crystal Reports (version 13, USA). These were used for auditing purposes.

\section{Additions to physical resources}

- Equipment for the clinic was obtained mostly through donations. We ensured that all rooms had tuning forks, patellar hammers and monofilament for testing the feet. A BMI scale was acquired. All consulting rooms had a functional ophthalmoscope, enabling accurate fundoscopy to be performed.

\section{The multidisciplinary team}

- Foot care is an integral part of diabetes management, as individuals with $\mathrm{DM}$ are 20 times more prone to lower limb amputations than non-diabetics. ${ }^{[13]}$ The podiatrist working at the local tertiary hospital was recruited to provide group patient education at the clinic while patients waited to see the clinicians, and also to consult individual patients with specific foot problems identified by the clinicians.

- Dietary changes are an important lifestyle modification that needs to be started as soon as a patient is diagnosed with DM and continued for life thereafter. ${ }^{[14]}$ Dietary patterns of patients of different ethnic groups need to be considered when dietary education is being given. After consultation with the dietetics department at Edendale Hospital it was agreed that dieticians would attend the clinic to provide group dietary education, and that all patients attending the clinic would be consulted individually at least once a year. Dietary education pamphlets in various languages were issued to patients as well.

- Patients were referred to the eye clinic for annual diabetes eye screening as per local diabetes guidelines. This screening is essential, as diabetic retinopathy is responsible for most cases of new-onset blindness in adults aged 20 - 74 years. ${ }^{[15]}$

\section{Issuing of glucometers to patients}

- SMBG has been shown to improve metabolic control in patients with DM. ${ }^{[16]}$ The majority $(71.2 \%)$ of the patients at our clinic had previously had no access to glucometers. ${ }^{[4]}$ An ongoing donation of glucometers was secured. All patients on any form of insulin therapy were given one, with education on its use and instructions on what to do in the event of hypo- or hyperglycaemia.

\section{Conclusions}

Patient education disseminated from all members of the multidisciplinary team and coupled with ongoing in-service training of clinicians and nurses is an integral component of this multifaceted approach to diabetes care. The ink-based stamp at the nurses' station ensures that every patient has vital clinical information measured and entered into the OP file. This information allows the clinician to assess for RBG, postural hypotension, urine dipstick findings such as proteinuria and evidence of urinary tract infections, raised BMI and waist-to-height ratios indicating obesity. The creation of the datasheet and its incorporation into the clinic ensures comprehensive and standardised management of all patients seen. The datasheet helps direct clinicians in approaching the complex entity of diabetes assessment and management. The computer program enables us to evaluate the baseline state of diabetes control in the clinic, and we will also be able to monitor trends in diabetes control there over future years. With improved clinic booking systems and referral criteria, the number of patients seen has been brought down to a manageable 30 40 a week. This allows clinicians to spend more time with patients, conducting a thorough diabetes history and clinical examination. 
The changes we have made in this resource-limited clinic could be adapted to other similar clinics in developing countries, as well as to district and regional hospitals. If necessary, datasheets could be completed at other health institutions and sent to a central regional hospital for capturing onto a customised computer program. Reports generated in this way could provide important information regarding diabetes control in these healthcare facilities.

$\mathrm{DM}$ is a complex disease entity that requires a holistic and multifaceted approach to ensure adequate control with minimal complications. Strategies like ours may help provide a blueprint for other healthcare facilities in developing countries to tackle the global pandemic of DM.

1. Galli L, Salpietro S, Pellicciotta G, et al. Risk of type 2 diabetes among HIV-infected and healthy subjects in Italy. Eur J Epidemiol 2012;27(8):657-665, DOI:10.1007/s10654-012-9707-5

2. Department of Health, South Africa. Annual Performance Plan 2012/13 - 2014/15. http://www.tbfacts. org/wp-content/uploads/2015/06/App2012-2014.pdf (accessed 1 November 2015).
.

3. Ng M, Fleming T, Robinson M, et al. Global, regional, and national prevalence of overweight and obesity in children and adults during 1980-2013: A systematic analysis for the Global Burden of Disease Study 2013. Lancet 2014;384(9945):766-781. DOI:10.1016/S0140-6736(14)60460-8

4. Pillay S, Aldous C, Mahomed F. Diabetic patients served at a regional level hospital: What is their clinical picture? J Endocrinol Metab Diabetes S Afr 2015;20(1):60-66. DOI:10.1080/16089677.2015.1030856

5. Amod A, Riback W. Diabetes guidelines and clinical practice: Is there a gap? The South African cohort of the International Diabetes Management Practices Study. J Endocrinol Metab Diabetes S Afr 2012;17(2):85-90. DOI:10.1080/22201009.2012.10872282
6. Igbojiaku OJ, Harbor OC, Ross A. Compliance with diabetes guidelines at a regional hospital in KwaZulu-Natal, South Africa. Afr J Prim Health Care Fam Med 2013;5(1). DOI:10.4102/phcfm. v5i1.447

7. Kirkman MS, Williams S, Caffrey H, et al. Impact of a program to improve adherence to diabetes guidelines by primary care physicians. Diabetes Care 2002;25(11):1946-1951. DOI:10.2337 guidelines by pring

8. Hetlevik I, Holmen J, Midthjell K. Treatment of diabetes mellitus - physicians' adherence to clinical guideline in Norway. Scand J Prim Health Care 1997;15(4):193-197. DOI:10.3109/02813439709035027 9. Pollard C, Bailey KA, Petitte T, et al. Electronic patient registries improve diabetes care and clinical Pollard C, Bailey KA, Petitte T, et al. Electronic patient registries improve diabetes care and clinical
outcomes in rural community health centers. J Rural Health 2009:25(1):77-84. DOI:10.1111/j.1748outcomes in rural
$0361.2009 .00202 \times$

10. Ricci-Cabello I, Ruiz-Perez I, Rojas-Garcia A, Pastor G, Goncalves DC. Improving diabetes care in rural areas: A systematic review and meta-analysis of quality improvement interventions in OECD countries. PLoS One 2013;8(12):e84464. DOI:10.1371/journal.pone.0084464

11. Pillay S, Lutge E, Aldous C. The burden of diabetes mellitus in KwaZulu-Natal's public sector: A 5-year perspective. S Afr Med J 2016;106(4):384-388. DOI:10.7196/SAMJ. 2016.v106i4.9920

12. Amod A, Motala A, Levitt N, et al. The 2012 SEMDSA guidelines for the management of type 2 diabetes. J Endocrinol Metab Diabetes S Afr 2012;17(1):S1-S94. DOI:10.1080/22201009.2012.10872277

13. Van Houtm WH, Lavery LA, Harkless LB. The impact of diabetes-related lower-extremity amputations in the Netherlands. J Diabetes Complications 1996;10(6):325-33. DOI:10.1016/1056-8727(95)00088-7

14. Andrews RC, Cooper AR, Montgomery AA, et al. Diet or diet plus physical activity versus usual care in patients with newly diagnosed type 2 diabetes: The Early ACTID randomised controlled trial. Lancet patients with newly diagnosed type 2 diabetes: The Early ACTID

15. Fong DS, Aiello L, Gardner TW, et al. Retinopathy in diabetes. Diabetes Care 2004;27(1):S84-S87. Fong DS, Aiello L, Gardner TW,
DOI:10.2337/diacare.27.2007.S84

16. Guerci $B$, Drouin $P$, Grange V, et al. Self-monitoring of blood glucose significantly improves metabolic control in patients with type 2 diabetes mellitus: The Auto-Surveillance Intervention Active (ASIA) study. Diabetes Metab 2003;29(6):587-594. DOI:10.1016/S1262-3636(07)70073-

Accepted 30 November 2015. 